Open Access

\title{
Neurostimulation for cognitive rehabilitation in stroke (NeuroCog): study protocol for a randomized controlled trial
}

\author{
Suellen Marinho Andrade ${ }^{1 *}$, Bernardino Fernández-Calvo ${ }^{1}$, Paulo Sérgio Boggio² ${ }^{2}$ Eliane Araújo de Oliveira ${ }^{3}$, \\ Lilze Franklim Gomes ${ }^{4}$, José Eudes Gomes Pinheiro Júnior ${ }^{5}$, Rafaela Martins Rodrigues ${ }^{4}$, \\ Natália Leandro de Almeida ${ }^{4}$, Gioconda Marla de Siqueira Moreira ${ }^{4}$ and Nelson Torro Alves ${ }^{1}$
}

\begin{abstract}
Background: Stroke patients may present severe cognitive impairments, primarily related to executive functions. Transcranial direct current stimulation has shown promising results, with neuromodulatory and neuroplastic effects. This study is a double-blind, sham-controlled clinical trial aiming to compare the long-term effects of stimulation in two different cognitive regions after a stroke.

Methods/Design: Sixty patients who suffer from chronic strokes will be randomized into one of four groups: dorsolateral prefrontal cortex, cingulo-opercular network, motor primary cortex and sham stimulation. Each group will receive transcranial direct current stimulation at an intensity of $2 \mathrm{~mA}$ for 20 minutes daily for 10 consecutive days. Patients will be assessed with a Dysexecutive Questionnaire, Semantic Fluency Test, categorical verbal fluency and Go-no go tests, Wechsler Adult Intelligence Scale, Rey Auditory-Verbal Learning Test, Letter Comparison and Pattern Comparison Tasks at baseline and after their tenth stimulation session. Those who achieve clinical improvement with neurostimulation will be invited to receive treatment for 12 months as part of a follow-up study.
\end{abstract}

Discussion: Long-term stimulation could be analyzed in regard to possible adaptive changes on plasticity after structural brain damage and if these changes are different in terms of clinical improvement when applied to two important cognitive centers.

Trials registration: Clinicaltrials.gov, NCT02315807. 9 December 2014.

Keywords: Transcranial direct current stimulation, Cognitive rehabilitation, Clinical trial

\section{Background}

Because cerebrovascular diseases are considered a major factor of mortality and morbidity in many parts of the world, new therapeutic methods have been proposed for the management of cognitive sequelae after a stroke. However, few studies have examined cognitive factors associated with treatment by neurostimulation after a stroke. More emphasis has been directed at functional improvement related to outcomes such as mobility, independence and daily living activities [1]. In

\footnotetext{
* Correspondence: suellenandrade@gmail.com

${ }^{1}$ Cognitive Neuroscience and Behavior Program, Department of Psychology, Federal University of Paraíba, João Pessoa, Brazil

Full list of author information is available at the end of the article
}

contrast, it is estimated that $75 \%$ of people will have losses in executive functions after a stroke. These difficulties cause problems in their abilities to perform dayto-day tasks and deal with other problems such as movement disorders, which are also derived from their brain injury [2].

Neurostimulation techniques such as transcranial magnetic stimulation (TMS) and transcranial direct current stimulation (tDCS) have shown promising results in this population [3]. Both techniques can lead to an improvement of lost skills/abilities, with virtually no side effects or discomfort for patients [4]. Regarding tDCS, there are numerous advantages compared to other non-invasive brain stimulation therapies, such as low cost, easy handling and high portability. During the
C Biomed Central

(c) 2015 Andrade et al. Open Access This article is distributed under the terms of the Creative Commons Attribution 4.0 International License (http://creativecommons.org/licenses/by/4.0/), which permits unrestricted use, distribution, and reproduction in any medium, provided you give appropriate credit to the original author(s) and the source, provide a link to the Creative Commons license, and indicate if changes were made. The Creative Commons Public Domain Dedication waiver (http://creativecommons.org/publicdomain/zero/1.0/) applies to the data made available in this article, unless otherwise stated. 
application of electric current, essential neurostimulatory effects are observed. After stopping tDCS application, neuroplastic effects can be observed [5].

With regard to the stimulation site of cognitive areas, it is not yet clear what role each area would play during the planning and execution of cognitive information [6]. Although the dorsolateral prefrontal cortex (dlPFC) is considered the main cognitive control area used in experiments with tDCS [7], neuroimaging techniques have contributed to identifying other "multiple functional neural networks," which may be related to processing or cognitive control categories [8]. A study involving mapping and interconnection of various regions showed that the frontoparietal network could serve as a "flexible hub" that would alter connectivity with other neural networks based on the specific task [9]. Other authors indicate that the parietal component seems to initiate and adjust the control, while the cingulo-opercular network (CON) provides a stable maintenance of the task, suggesting connections between two core networks and other weaker long-range connections between components [10].

In this respect, some aspects of cognition can be broadly generalized, and there is no agreed upon list of all components comprising executive functions (as an example). This results in discrepancies between the studies concerning which the factors should be related [11, 12].

To address the theoretical gaps, we propose that the cingulo-opercular network could serve as an alternative locus of stimulation for cognitive rehabilitation of these patients. Combined with this, this work represents the first controlled clinical trial involving the application of tDCS on stroke. It will include a follow-up of 12 months, making it possible to investigate not only the effects that occur in the short term, but also those that last for a longer period after neurostimulation. In a recent study that evaluated cognitive problems after strokes in young patients ( $<50$ years), it was found that "mental slowness" occurs up to 10 years after brain injury, and so it is necessary to assess the presence of cognitive deficits after a long period of follow-up [13].

Thus, the objective of this study is to 1) evaluate the efficacy of tDCS on two different networks, the frontoparietal (specifically in the dIPFC) and the cinguloopercular (anterior insula/frontal operculum), comparing them with each other for cognitive rehabilitation after a chronic stroke; 2) check whether there are differences between the benefits achieved by stimulation of these two cortical areas and how big these differences are; and 3) present the study protocol, the previous results of the clinical trial, ensuring adherence and compliance with the guidelines previously proposed.

\section{Methods/Design}

\section{Design}

A clinical double-blind, randomized, controlled trial will be performed involving patients with chronic stroke, randomly assigned to four groups: Group 1) active tDCS in fronto-parietal region (dlPFC); Group 2) active tDCS in the cingulo-opercular region-CON (anterior insula/ frontal operculum); Group 3) active tDCS in the primary motor cortex (M1); and Group 4) sham tDCS in M1. This region was used as a place of active and placebo control.

Initially, all participants will undergo 10 consecutive daily sessions of neurostimulation (excluding weekends). Evaluations will be conducted pre- and post-test (T0 and T1, respectively). After 1 month, the participants will be checked to see who attained clinical improvement with treatment: that is, those patients who had a final score (T2) on the Dysexecutive Questionnaire (DEX) greater than at least 2.77 standard error of measurement compared from their baseline/initial testing [14]. Those who have an adequate response will be invited to receive treatment for 12 months. In this period of follow-up, the stimulation sessions will be applied twice monthly, for 5 consecutive days. This model is similar to another that has already been applied/used by other authors with positive results in a study involving tDCS for treatment of depression in order to assess whether the benefits of regularly performed neurostimulation continue in the long term [15]. Adverse effects will be computed periodically to prevent possible deleterious effects (see Fig. 1, which demonstrates the flow of the study). Previous studies have demonstrated that tDCS induces minimal discomfort sensations, such as mild tingling and itching sensations under the electrodes, predominantly in the first few seconds of the tDCS $[16,17]$.

The method of randomization will be 1: 1: 1: 1 , with interchange between blocks generated by an online program (random.org). Concealed allocation will be used with numbered, opaque-sealed sequential envelopes so that the person responsible for allocation will not be in contact with patients or with the work of others. All examiners will be blind to the type of treatment the patient is receiving (site of stimulation) and other assessments that will be carried out. The efficiency of the masking/ blinding mechanism will be evaluated at the time of the last interview with the evaluators. They will state what region they believe the patient received stimulation in. Patients will also be blinded with a masking evaluation carried out on the last post-test where they will try to guess in which region stimulation was applied: the main (dIPFC) or secondary area (CON) of cognitive control, the motor control region (M1) or sham stimulation.

Attrition will be considered when a) three alternating or two consecutive absences; b) presence of cognitive 


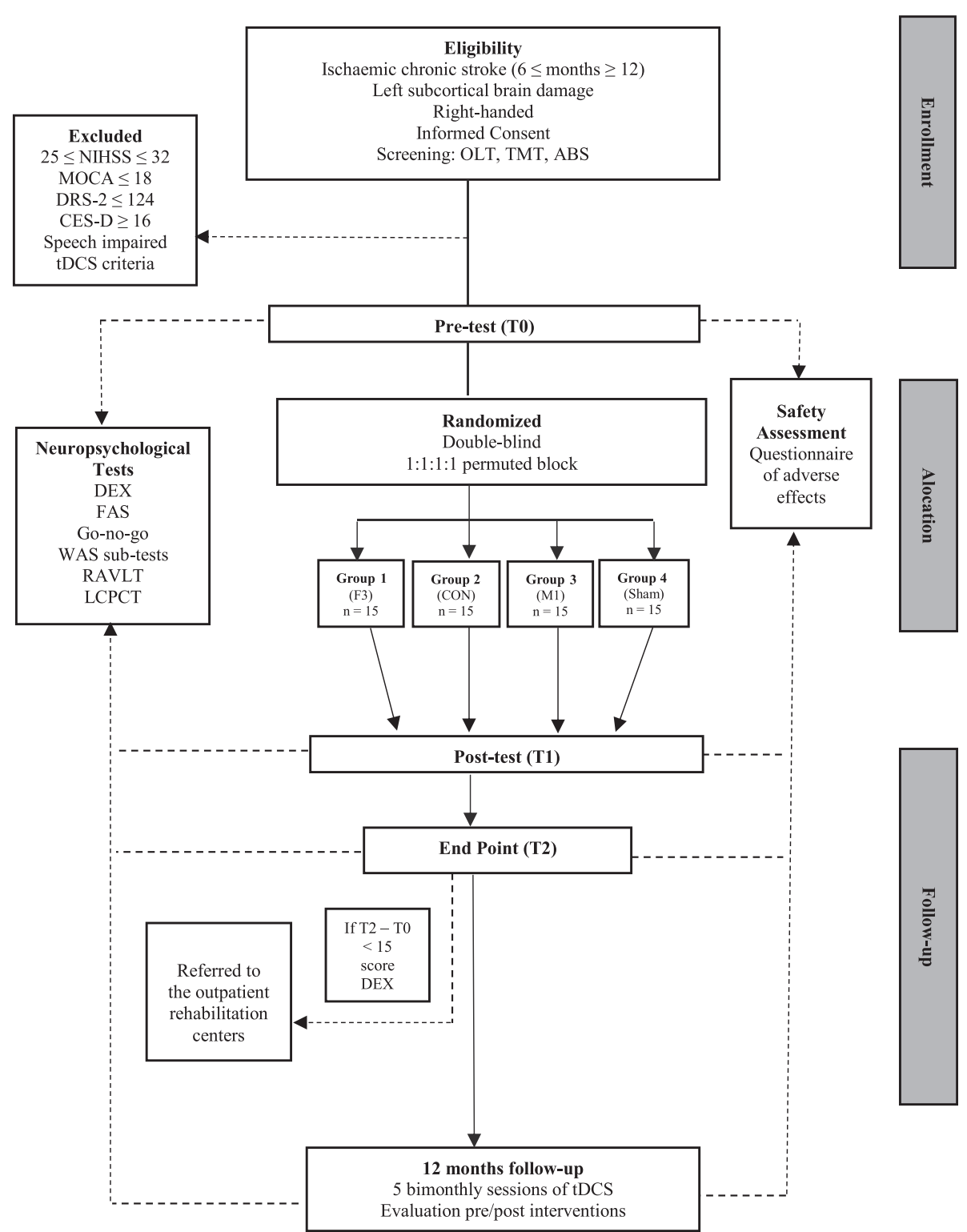

Fig. 1 Flow chart of study. ASB: Assessment of Stroke and other Brain Damage; CES-D: Center for Epidemiological Studies-Depression; CON: cingulo-opercular network; DEX: Dysexecutive Questionnaire; dIPFC: dorsolateral prefrontal cortex; FAS: Semantic Fluency Test; DRS-2: Dementia Rating Scale-2; LCPCT: Letter Comparison and Pattern Comparison Tasks; M1: primary motor cortex; MOCA: Montreal Cognitive Assessment; NIHSS: National Institute of Health Stroke Scale; OLT: Object Learning Test; RAVLT: Rey Auditory-Verbal Learning Test; tDCS: transcranial direct current stimulation; TMT: Trail Making Test; WAIS: Wechsler Adult Intelligence Scale

comorbidities throughout the trial; c) loss during followup. There will be adherence strategies administered such as being able to make up an absence the following week, and offering flexible schedules and frequent contact through phone calls confirming dates of procedures and reinforcing the importance of treatment compliance.

The study was approved by the Health Sciences Centre Ethical Committee (\#30163714.0.0000.5188) and is governed according to the principles of the Declaration of Helsinki. To conduct the study, the informed consent of each participating patient will be collected. The protocol follows all the recommendations of the Standard Protocol Items: Recommendations for Interventional Trials Statement (SPIRIT) 2013 [18].

\section{Participants}

Sixty right-handed patients will be selected, aged between 18 and 60 years, who have been diagnosed with stroke in the left subcortical ischemic of the middle cerebral artery territory; according to the International Classification of Diseases (ICD-10) and whose data have been obtained by computerized tomography or magnetic 
resonance. The occurrence of the injury within a 6 to 12 month period will be considered chronic [19]. Only those patients referred for cognitive rehabilitation based on the clinical diagnosis by the treatment team, including interviews and neuropsychological testing [20], will be included in the clinical study. The neuropsychological screen/testing will consist of the following tests [21]. The Object Learning Test [22] will be used to assess learning and short-time memory for visually presented material. The cutoff between normal and pathologic cognitive functioning is 36 points for patients $\leq 64$ years. The Trail Making Test A [23], which will be used to assess psychomotor speed and attention, has a cutoff for normal and impaired cognitive function equivalent to 60 s. An Assessment of Stroke and other Brain Damage [24] will be used to evaluate motor apraxia, rational apraxia and visual-spatial ability. The present study will be set at 65 points for motor apraxia (lower quartile), 14 points for rational apraxia (full score), and 25 points for visual-spatial ability (lower quartile).

Exclusion criteria are as follows: inability to perform the tests due to losses in production or understanding speech; severe clinical status, assessed by the National Institute of Health Stroke Scale (NIHSS) $\geq 16$ points [25]; severe cognitive comorbidities, diagnosed according to clinical evaluation and according to the Diagnostic and Statistical Manual of Mental Disorders, 4th. Edition (DSM-IV) [26], and supplemented by the following instruments: Montreal Cognitive Assessment (MOCA) $\leq 18$ points [27], Dementia Rating Scale-2 (DRS-2) $\leq 124$ points [28] , and the Center for Epidemiological Studies - Depression (CES-D) $\geq 16$ points [29]. In addition, exclusion criteria for the use of cortical stimulation will be applied: a) use of drugs stimulating central nervous system activity; b) patients with implanted metallic or electronic devices; c) a cardiac pacemaker; d) patients who experience seizures/ convulsions; and e) pregnancy.

\section{Intervention}

A constant current stimulator (TCT Research Limited) will be used using electrodes of $5 \times 5 \mathrm{~cm}^{2}$ embedded in saline $(0.9 \% \mathrm{NaCl})$ and application of $2 \mathrm{~mA}$ current for 20 minutes. The protocol is identical for placebo stimulation, but the current will stop after 30 seconds from the start of stimulation, a blinding method used in several previous studies $[1,15]$.

The positioning of the electrodes follows the 10-20 electroencephalogram (EEG) system model. The cathode will be positioned in the right supra-orbital region (contralateral to vascular injury), while the anode will have the following provision in the left hemisphere: group 1 (dIPFC), F3 position [30]; group 2, cingulo-opercular network $(\mathrm{CON})$-anterior insula/frontal operculum, the intersection point between $\mathrm{T} 3-\mathrm{Fz}$ and $\mathrm{F} 7-\mathrm{Cz}$ [31, 32]; and groups 3 and 4, M1 position [33].

All experiments will be conducted at the same time of day (afternoon shift), including stimulation and evaluation, to avoid possible circadian cycle influences.

\section{Procedures}

The primary outcome of the study will be the Dysexecutive Questionnaire (DEX) [34], composed of 20 subtests, standardized, with total score ranging from 0 to 80 points, applied to the patient and a family member. The issues involve disturbances in executive functions such as emotional or personality, motivational, behavioral and cognitive changes. It is an instrument with ecological validity confirmed for patients with brain injuries and may provide important information on executive functions with implications for cognitive rehabilitation [35].

Secondary outcomes will be: the Semantic Fluency Test (FAS), which assesses the recall of words that begin with a certain letter and category fluency for the generation of words in a semantic class [36]; Go-no go, which tests selfmonitoring, inhibition, initiation and cognitive flexibility [37]; Wechsler Intelligence Scale for Adults (WAIS), in the subtests of digit symbol coding, matrix reasoning, comprehension and letter-number sequencing for evaluation of executive functions [38]; Rey Auditory Verbal Learning (RAVLT) test that measures recent memory, learning, interference, retention and recognition memory [39]; Letter Comparison and Pattern Comparison Tasks [40].

\section{Safety}

To control adverse effects, reports of patients with feelings/sensations of itching, tingling, burning, headache or other discomfort (1 none, 2 mild, 3 moderate, or 4 strong) will be recorded, along with whether this effect could be related to stimulation on a Likert scale; 1 (no relation) to 5 (strongly related).

If any damage/injury occurs to the participant, he or she will be offered medical assistance/treatment, physiotherapy and psychological care without any onus or cost to the participant.

\section{Statistical analysis}

To determine the number of participants in each group, we will apply the proposed criteria for clinical trials. As observed in previous studies using the DEX for cognitive evaluation, we found that the average obtained by individuals with cognitive decline (without presence of dementia) undergoing rehabilitation protocols lies in a range of 15 to 17 points, with a deviation-pattern of 5.0 to 8.0 points [41-43]. The minimal importance difference will be considered based on the variation of the standard error of measurement approach, considering 1 for a small effect, 1.96 for a moderate and 2.77 for a 
large effect [14]. From these criteria, considering $90 \%$ power and taking into account possible dropouts, we calculate a final sample size of 90 participants. A significance level of $P<0.05$ will be considered.

An analysis of intention to treat will be used, and sensitivity analysis will be performed with different allocation procedures to verify the strength of the data. The best strategy resulting from the comparison of the following methods will be chosen: last observation carried forward, complete-case analysis, likelihood-based methods and multiple imputation.

For the primary endpoint, we will use a mixed linear model. We will model the change in cognitive performance according to the DEX using the covariates of time, group and interaction between treatment and time. The covariance with repeated measures and for each patient will also be measured. For secondary outcomes, repeated measures analysis of variance (ANOVA) will be used in which the dependent variable is the performance in each cognitive test and the independent variables are group (dlPFC, CON, M1, or sham), treatment time (baseline/ initiation, week 2 and follow-up) and interaction group $\times$ treatment time. If necessary, post-hoc comparisons using Bonferroni correction will be made.

Deleterious and adverse cognitive effects will be calculated in terms of the proportion in each group and in each period, and will be analyzed by the Fisher exact test.

\section{Discussion}

The primary objective of the study is to determine whether stimulation of the dlPFC and CON affects cognition in patients after a stroke. In addition, we seek to examine whether these effects are similar to each other and what is the clinical significance of the identified changes. Although studies with noninvasive stimulation focused on patients with cognitive dysfunction are important, completing a long-term follow up of participants who suffer strokes is a possibility for new advances in terms of prognosis. Traditionally, they would only have access to motor rehabilitation.

Some work has been published in order to explore the role that these two dIPFC and CON networks play [44]. However, clinical evidence related to the function of these two areas in terms of strategies for decision-making and other real life situations has been compromised [45]. In order to compare the effects of neurostimulation at these two sites, we propose to identify recovery markers through standardized assessment protocols.

In general, the strengths of our study include the following: 1) proposing a new approach of different stimulation from the classical cognitive approach after a stroke; 2) conduct longitudinal monitoring of responses to therapy, measuring potential neuroplastic benefits; 3) employing rehabilitation that is easy to use, low cost, safe and feasible; and 4) characterize cognitive markers as predictors of response.

Nevertheless, we can cite the insertion of chronic patients as a constraint, which limits the generalization to other phases of the stroke. Because there are cognitive and sensorimotor differences in the various phases of a stroke, it was decided to choose one of them, for greater control of the data. This, however, does not preclude the conduct of future studies that compare patients in different stages in order to know at what time after the injury stimulation could be more effective. Furthermore, the treatment will be performed in the laboratory, which can result in losses or withdrawals by participants. To minimize these effects, adherence strategies will be employed (direct and frequent contact with patients, scheduling flexibility and reinforcement of the importance of care continuity).

Another important aspect is that during the repeated measures application of neuropsychological tests, ceiling effects may occur, which will reduce the longer the interval between assessments; conversely, there will be a greater risk of changes in the characteristic being measured. In this study, the constructs analyzed did not express significant volatility over time, and the assessment range was based on standardization applied in previous studies involving neurostimulation and cognitive assessment $[33,46]$. Thus, our study provides important implications for rehabilitation, both in the theoretical aspect to support new trials and also in a clinical setting to provide evidence to be applied in treating patients.

\section{Trial status}

Recruitment and interventions are in progress.

\section{Abbreviations}

ANOVA: analysis of variance; CES-D: Center for Epidemiological Studies Depression; CON: cingulo-opercular network; DRS-2: Dementia Rating Scale-2; DSM-IV: Diagnostic and Statistical Manual of Mental Disorders; dIPFC: dorsolateral prefrontal cortex; DEX: Dysexecutive Questionnaire; EEG: electroencephalogram; ICD-10: International Classification of Diseases; MOCA: Montreal Cognitive Assessment; NIHSS: National Institute of Health Stroke Scale; M1: primary motor cortex; RAVLT: Rey Auditory Verbal Learning; FAS: semantic fluency test; SPIRIT: Standard Protocol Items: Recommendations for Interventional Trials; tDCS: transcranial direct current stimulation; TMS: transcranial magnetic stimulation; WAIS: Wechsler Intelligence Scale for Adults.

\section{Competing interests}

The authors declare that they have no competing interests.

\section{Authors' contributions}

SMA, BFC, PSB, and NTA led the design, implementation of this research protocol and drafted the manuscript. LFG, JEGPJ, RMR, NLdA, and GMS conducted the research (hands-on conduct of experiments and data collection). EAdO participated in the design, coordination and the setup for the laboratory. All authors read and approved the final manuscript.

\section{Acknowledgements}

The authors are grateful for assistance provided by the Laboratory of Cognitive Neuroscience and Behavior. This trial is funded by TCT Research Limited. This company has had no influence on the study design, collection, 
management, and interpretation of data; writing the report; or submitting the report for publication.

\section{Author details}

${ }^{1}$ Cognitive Neuroscience and Behavior Program, Department of Psychology, Federal University of Paraíba, João Pessoa, Brazil. ${ }^{2}$ Cognitive Neuroscience Laboratory and Developmental Disorders Program, Mackenzie Presbyterian University, São Paulo, SP, Brazil. ${ }^{3}$ Center for Research in Human Movement Sciences, Federal University of Paraíba, João Pessoa, PB, Brazil. ${ }^{4}$ Perception, Neurosciences and Behavior Laboratory, Federal University of Paraíba, João Pessoa, PB, Brazil. ${ }^{5}$ Department of Sciences of Religions, NOUS Group, Federal University of Paraíbas, João Pessoa, PB, Brazil.

\section{Received: 26 January 2015 Accepted: 7 September 2015}

\section{Published online: 29 September 2015}

\section{References}

1. Khedr EM, Shawky OA, El-Hammady DH, Rothwell JC, Darwish ES, Mostafa $\mathrm{OM}$, et al. Effect of anodal versus cathodal transcranial direct current stimulation on stroke rehabilitation: a pilot randomized controlled trial. Neurorehabil Neural Repair. 2013;27:592-601.

2. Chung CSY, Pollock A, Campbell T, Durward BR, Hagen S. Cognitive rehabilitation for executive dysfunction in adults with stroke or other adult non-progressive acquired brain damage. Cochrane Database Syst Rev. 2013:4:CD008391.

3. Thibaut A, Chatelle C, Gosseries O, Laureys S, Bruno M-A. Transcranial direct current stimulation: a new tool for neurostimulation. Rev Neurol (Paris). 2013;169:108-20.

4. Nahas Z, Kozel FA, Li X, Anderson B, George MS. Left prefrontal transcranial magnetic stimulation (TMS) treatment of depression in bipolar affective disorder: a pilot study of acute safety and efficacy. Bipolar Disord. 2003;5:40-7.

5. Brunoni AR, Nitsche MA, Bolognini N, Bikson M, Wagner T, Merabet L, et al. Clinical research with transcranial direct current stimulation (tDCS): challenges and future directions. Brain Stimul. 2012;5:175-95.

6. Cocchi L, Zalesky A, Fornito A, Mattingley JB. Dynamic cooperation and competition between brain systems during cognitive control. Trends Cogn Sci. 2013;17:493-501.

7. Beeli G, Koeneke S, Gasser K, Jancke L. Brain stimulation modulates driving behavior. Behav Brain Funct. 2008:4:34.

8. Power JD, Cohen AL, Nelson SM, Wig GS, Barnes KA, Church JA, et al. Functional network organization of the human brain. Neuron. 2011;72:665-78.

9. Zanto TP, Gazzaley A. Fronto-parietal network: flexible hub of cognitive control. Trends Cogn Sci. 2013;17:602-3.

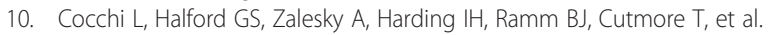
Complexity in relational processing predicts changes in functional brain network dynamics. Cereb Cortex. 2014;24:2283-96.

11. Dosenbach NUF, Fair DA, Cohen AL, Schlaggar BL, Petersen SE. A dual-networks architecture of top-down control. Trends Cogn Sci (Regul Ed). 2008;12:99-105.

12. Nomura EM, Gratton C, Visser RM, Kayser A, Perez F, D'Esposito M. Double dissociation of two cognitive control networks in patients with focal brain lesions. Proc Natl Acad Sci U S A. 2010;107:12017-22.

13. De Bruijn MAAM, Synhaeve NE, van Rijsbergen MWA, de Leeuw F-E, Jansen BPW, de Kort PLM. Long-term cognitive outcome of ischaemic stroke in young adults. Cerebrovasc Dis. 2014;37:376-81.

14. Wyrwich KW, Tierney WM, Wolinsky FD. Further evidence supporting an SEM-based criterion for identifying meaningful intra-individual changes in health-related quality of life. J Clin Epidemiol. 1999;52:861-73.

15. Valiengo L, Benseñor IM, Goulart AC, de Oliveira JF, Zanao TA, Boggio PS, et al. The sertraline versus electrical current therapy for treating depression clinical study (select-TDCS): results of the crossover and follow-up phases. Depress Anxiety. 2013;30:646-53.

16. Fertonani A, Ferrari C, Mninussi C. What do you feel if I apply transcranial electric stimulation? Safety, sensations and secondary induced effects. Clin Neurophysiol. 2015; doi: 10.1016/j.clinph.2015.03.015. [Epub ahead of print].

17. Nitsche MA, Liebetanz D, Lang N, Antal A, Tergau F, Paulus W. Safety criteria for transcranial direct current stimulation (tDCS) in humans. Clin Neurophysiol. 2003;114(11):2220-2.

18. Chan A-W, Tetzlaff JM, Altman DG, Laupacis A, Gøtzsche PC, Krleža-Jerić K, et al. SPIRIT 2013 Statement: Defining standard protocol items for clinical trials. Ann Intern Med. 2013;158:200-7.
19. Elsner B, Kugler J, Pohl M, Mehrholz J. Transcranial direct current stimulation (tDCS) for improving function and activities of daily living in patients after stroke. Cochrane Database Syst Rev. 2013;11:CD009645.

20. Winkens I, Van Heugten CM, Wade DT, Habets EJ, Fasotti L. Efficacy of time pressure management in stroke patients with slowed information processing: a randomized controlled trial. Arch Phys Med Rehabil. 2009;90:1672-9.

21. Fure B, Bruun Wyller T, Engedal $K$, Thommessen B. Cognitive impairments in acute lacunar stroke. Acta Neurol Scand. 2006;114:17-22.

22. Kendrick DC. The Kendrick Battery of tests: theoretical assumptions and clinical uses. Br J Soc Clin Psychol. 1972;11:373-86.

23. Reitan RM. The relation of the trail making test to organic brain damage. J Consult Psychol. 1955;19:393-4.

24. Bernspång B, Fugl-Meyer AR, Viitanen M. Perceptual function in the elderly and after stroke. Scand J Caring Sci. 1988;2:75-9.

25. Brott T, Adams HP, Olinger CP, Marler JR, Barsan WG, Biller J, et al. Measurements of acute cerebral infarction: a clinical examination scale. Stroke. 1989;20:864-70.

26. American Psychiatric Association. Diagnostic and statistical manual of mental disorders. Washington: American Psychiatric Association; 1987.

27. Nasreddine ZS, Phillips NA, Bédirian V, Charbonneau S, Whitehead V, Collin I, et al. The montreal cognitive assessment, MoCA: a brief screening tool for mild cognitive impairment. J Am Geriatr Soc. 2005:53:695-9.

28. Mattis S. Dementia rating scale. Professional manual. Florida: Psychological Assessment Resources; 1988.

29. Radloff LS. The CES-D scale: a self-report depression scale for research in the general population. Appl Psychol Meas. 1977;1:385-401

30. Foerster A, Rocha S, Wiesiolek C, Chagas AP, Machado G, Silva E, et al. Sitespecific effects of mental practice combined with transcranial direct current stimulation on motor learning. Eur J Neurosci. 2013;37:786-94.

31. Monti A, Cogiamanian F, Marceglia S, Ferrucci R, Mameli F, Mrakic-Sposta S, et al. Improved naming after transcranial direct current stimulation in aphasia. J Neurol Neurosurg Psychiatr. 2008;79:451-3.

32. Cattaneo Z, Pisoni A, Papagno C. Transcranial direct current stimulation over Broca's region improves phonemic and semantic fluency in healthy individuals. Neuroscience. 2011;183:64-70.

33. Boggio PS, Ferrucci R, Rigonatti SP, Covre P, Nitsche M, Pascual-Leone A, et al. Effects of transcranial direct current stimulation on working memory in patients with Parkinson's disease. J Neurol Sci. 2006;249:31-8.

34. Wilson BA, Alderman N, Burgess PW. Behavioural assessment of the dysexecutive syndrome. Bury St. Edmund: Thames Valley Test Company; 1996.

35. Emmanouel A, Mouza E, Kessels RPC, Fasotti L. Validity of the Dysexecutive Questionnaire (DEX). Ratings by patients with brain injury and their therapists. Brain Inj. 2014;28:1581-9.

36. Troyer AK. Normative data for clustering and switching on verbal fluency tasks. J Clin Exp Neuropsychol. 2000;22:370-8.

37. Rubia K, Russell T, Overmeyer S, Brammer MJ, Bullmore ET, Sharma T, et al. Mapping motor inhibition: conjunctive brain activations across different versions of go/no-go and stop tasks. Neuroimage. 2001;13:250-61.

38. Wechsler D. Wechsler adult intelligence scale-III. Nova York: Psychological Corporation; 1997.

39. Rey A. L'Examen clinique en psychologie. Paris: Press Universitaire de France; 1958.

40. Salthouse TA, Kersten AW. Decomposing adult age differences in symbol arithmetic. Mem Cognit. 1993;21:699-710.

41. Manly T, Hawkins K, Evans J, Woldt K, Robertson IH. Rehabilitation of executive function: facilitation of effective goal management on complex tasks using periodic auditory alerts. Neuropsychologia. 2002;40:271-81.

42. Levine B, Stuss DT, Winocur G, Binns MA, Fahy L, Mandic M, et al. Cognitive rehabilitation in the elderly: effects on strategic behavior in relation to goal management. J Int Neuropsychol Soc. 2007;13:143-52.

43. Schweizer TA, Levine B, Rewilak D, O'Connor C, Turner G, Alexander MP, et al. Rehabilitation of executive functioning after focal damage to the cerebellum. Neurorehabil Neural Repair. 2008;22:72-7.

44. Rolls ET, Hornak J, Wade D, McGrath J. Emotion-related learning in patients with social and emotional changes associated with frontal lobe damage. J Neurol Neurosurg Psychiatr. 1994;57:1518-24.

45. Rogers RD, Owen AM, Middleton HC, Williams EJ, Pickard JD, Sahakian BJ, et al. Choosing between small, likely rewards and large, unlikely 
rewards activates inferior and orbital prefrontal cortex. J Neurosci. 1999;19:9029-38.

46. Brunoni AR, Valiengo L, Baccaro A, Zanão TA, de Oliveira JF, Goulart A, et al. The sertraline vs. electrical current therapy for treating depression clinical study: results from a factorial, randomized, controlled trial. JAMA Psychiatry. 2013;70:383-91.

Submit your next manuscript to BioMed Central and take full advantage of:

- Convenient online submission

- Thorough peer review

- No space constraints or color figure charges

- Immediate publication on acceptance

- Inclusion in PubMed, CAS, Scopus and Google Scholar

- Research which is freely available for redistribution 